\title{
The Zabrze'18 protocol is a feasible option to reduce the number of endomyocardial biopsies after heart transplantation
}

\author{
Grzegorz M. Kubiak, Radosław Kwieciński, Michał Zakliczyński, Piotr Przybyłowski, Michał O. Zembala
}

Department of Cardiac Surgery and Transplantology, SMDZ in Zabrze, Medical University of Silesia in Katowice, Silesian Centre for Heart Disease, Zabrze, Poland

Adv Interv Cardiol 2019; 15, 3 (57): 368-370

DOI: https://doi.org/10.5114/aic.2019.87895

Limited diagnostic yield of endomyocardial biopsy after heart transplantation

Endomyocardial biopsy (EMB) is the method of choice to assess the potential rejection episodes in post-transplant heart recipients [1]. Since it is an invasive procedure, it is associated with the risk of complications. Therefore, many attempts have been made to monitor the risk of potential rejection in a non-invasive manner. Among them, IMAGE and the CARGO gene-expression profiling protocols ultimately proved to be promising. Nevertheless, in most of the heart transplant centers across the world, EMB remained the gold standard to monitor rejection episodes. Moreover, the majority of the institutions developed local regimens of patient surveillance after heart transplantation (HTx). They can be divided into two main groups, depending on the general assumptions. The first is focused on the high number of EMBs performed according to a routine schedule (routine surveillance $E M B$ - rsEMB), which in theory enables the diagnosis and treatment of acute cellular rejection (ACR) episodes before the development of clinical signs of rejection. The second concept focuses on clinical symptoms that are believed to trigger $E M B$, which is performed in case of ACR suspicion - clinically driven EMB (CdEMB). Since the diagnostic yield of rSEMB is limited, which means that the majority of the results are unable to prove rejection, it has been postulated to decrease the number of routinely performed EMBs [2].

\section{Possible short- and long-term complications of EMB - from experimental to routine practice}

EMBs - were first performed by the Japanese cardiac surgeons Sakakibara and Konno in 1962, with the latter being considered the inventor of the method [3]. Likewise, EMB was introduced to clinical practice by Caves and colleagues in 1974 [4]. Interestingly, Japanese scientists made a significant contribution to the development and improvement of this technique. Nevertheless, due to medical-legislative reasons the national transplantation program in Japan for many years stayed below the national demands and possibilities. It should be emphasized that EMB, although widely characterized in the literature as a relatively safe technique with only a few unfavorable outcomes, may be associated with either acute or delayed complications - its frequency varies between $3 \%$ and $6 \%[5,6]$. Right ventricle perforation with the subsequent pericardial tamponade (0.5-2.6\%) pneumothorax (1\%), iatrogenic puncture of arteries (2\%), nerve paresis $(0.1 \%)$, hematomas $(0.4 \%)$ and peripheral arterial to venous fistulas $(0.1 \%)$ are to be enumerated among acute complications [6, 7]. Time-delayed complications consist of local bleeding (0.4\%), mechanical impairment of tricuspid valve $(25 \%)$ and pericardial effusion with delayed tamponade $(3 \%)[5,7,8]$.

\section{Technical aspects of EMB - now and then}

Historically, EMB was performed percutaneously via the supraclavicular approach to either the right or the left subclavian vein. A 9-French ( $\mathrm{Fr}$ ) sheath was introduced using Seldinger's technique; subsequently, a dedicated curved 7-Fr catheter was placed in the proximity of the interventricular septum. Then, the $7 \mathrm{Fr}$ bioptome was introduced through the sheath to obtain tissue specimens for the histopathological assessment. Techniques currently used focus on safety-associated issues - ultrasound examination before acquiring vascular access,

\section{Corresponding author:}

Grzegorz M. Kubiak MD, PhD, Department of Cardiac Surgery and Transplantology, SMDZ, Medical University of Silesia, Silesian Centre for Heart Disease, 9 Curie-Skłodowska St, 41-800 Zabrze, Poland, phone: +48 3237338 57, e-mail: gkubiak@sccs.pl

Received: 22.03.2019, accepted: 8.04.2019. 
flexible forceps with individually adapted curvatures, smaller sheaths to prevent vascular trauma, utilization of sheath-less techniques, etc. The approach above, including simultaneous ultrasound-guided puncture of great veins ("in play mode"), is associated with a reduced number of serious local complications such as hematomas and/or bleeding.

\section{Accessible clinical modalities to reduce the number of EMBs}

Different institutions across the world use different biopsy regimens that are based on long-lasting experience in the field of transplantology. The number of rsEMBs may vary between two and fifteen, depending on the institutional experience. It is important to underline that the number of routinely performed EMBs must not be addressed without the connection between immunosuppressive treatment. It is believed that the induction with aggressive immunosuppression prevents the occurrence of rejection episodes, which have a huge impact on prognosis. Treatment protocols currently used for induction therapy consist of a polyclonal anti-lymphocyte antibody or an anti-interleukin 2 (IL-2) monoclonal antibody. Rabbit anti-thymocyte globulin (rATG) is a T-cell-depleting antibody therapy used either as induction of immunosuppression during the acute phase of the transplantation or in the treatment of the glucocorticoid-resistant acute rejection episodes [9]. Since rATG decreases the number lymphocytes, its use is associated with the potential in- crease of life-threatening complications. Firstly, it is associated with the allergy phenomenon; therefore, it should be administered with anti-allergic agents. Secondly, it is crucial to underline the raised patient's vulnerability to infection, especially given at the initial phase after HTx. Among these, rapidly developing sepsis remains a major threat impairing in-hospital mortality. Last but not least, rATG is associated with the raised incidence of lymphoproliferative disorders. The alternatively used IL-2 (basiliximab) inhibits the proliferation of T-cells resulting from allograft antigen stimulation without a significant effect on the resting T-cells. Its different mechanism of action offers more selective immunosuppression. On the other hand, according to recent reports, it is associated with reduced 5-year survival after HTx as compared to rATG [10]. From the clinical point of view, the rATG activity is monitored via the CD3 count in the serum blood. In this phase the treatment brings to mind the phenomenon of the two-edged sword - on one side the patient may suffer from acute rejection while on the other he might experience a serious, generalized infection. Since the rate of biopsy-proven rejections after rATG induction should by definition be less frequent, there exists a growing tendency towards lowering the number of rsEMBs. Additionally, this conception is supported by the growing experience that, during the regimen of the immunosuppression containing adequately performed rATG therapy, the EMBs could be uneventfully omitted, especially during the first 4 weeks after HTx. It is noteworthy that some experi-

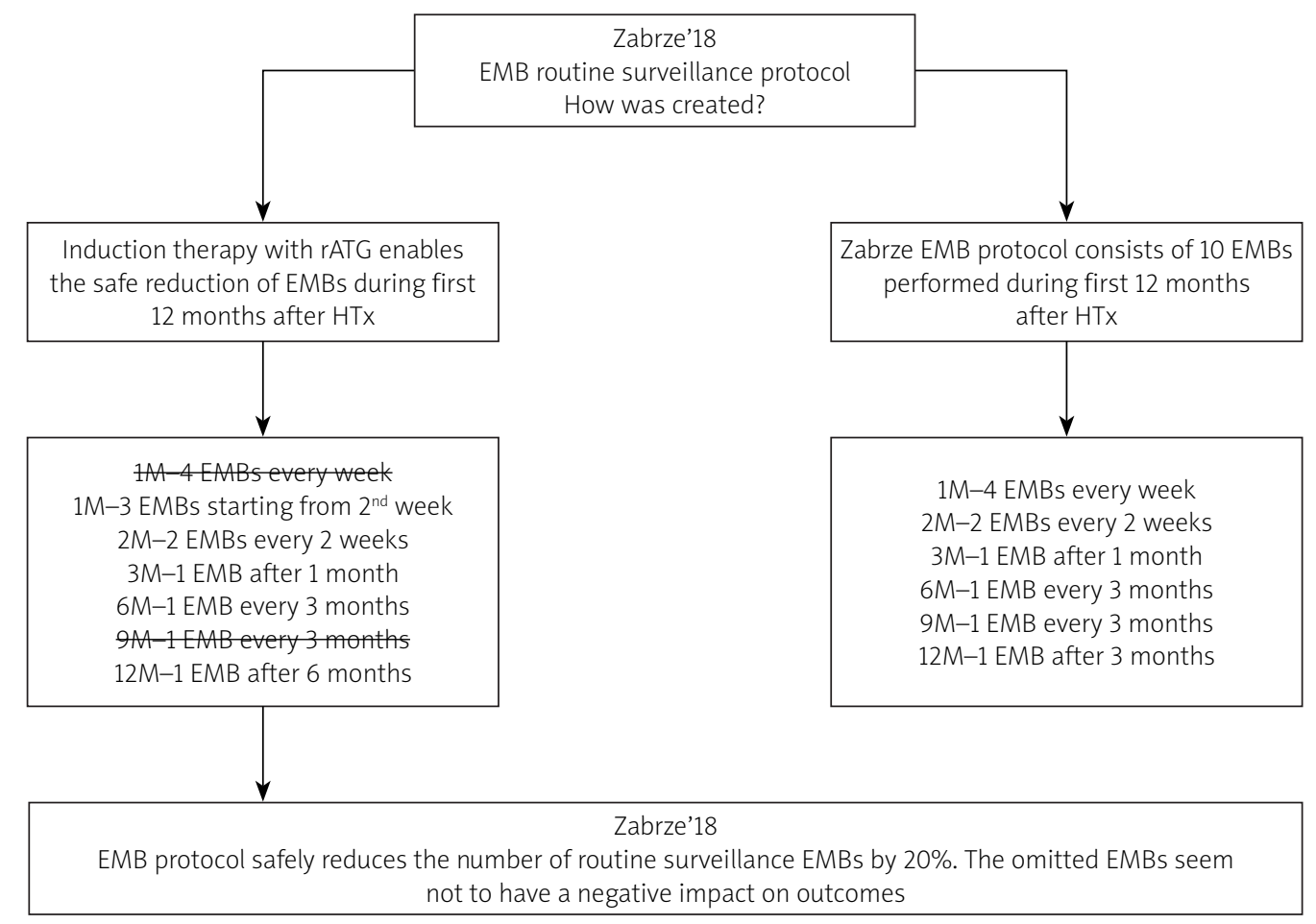

Figure 1. Zabrze'18 endomyocardial biopsy routine surveillance protocol

EMB - endomyocardial biopsy, $r A T G$ - rabbit anti-thymocyte globulin, 1-12M - one to twelve months, HTx - heart transplantation. 
enced centers limit the executed routine biopsies, in the first year after transplantation, to two performed in the third week and the twelfth month after the procedure.

We predefined the group of heart recipients presenting the highest potential to improve outcomes with the novel induction regimen. We focused on the immunological status and comorbidities prior to HTx in order to perform an individually tailored immunosuppression consisting of rATG. Inclusion criteria would be: informed written consent, age 18-65 years and panel reactive antibody score over ten percent. Exclusion criteria would be: bridge therapy with mechanical circulatory support prior to HTx, glomerular filtration rate less than $45 \mathrm{ml} /$ $\mathrm{min} / 1.73 \mathrm{~m}^{2}$ and the absence of active infection.

\section{Current implications for clinical practice}

With the continuously growing number of potential HTx recipients, modern transplantation programs are changing their attitude towards difficult donor-recipient pairs. There is a rising acceptance of transplantation in older patients with many comorbidities and bridged with assist devices. Moreover, the advanced age of the donors, especially given in Europe, is responsible for many new challenges occurring in the early post-transplant period. These phenomena reflect the sociological changes of the population of many Central and Eastern European countries. Zabrze, being a leading heart and lung transplantation center in Poland, has performed 76 heart and 27 lung transplantations this year. This cutting-edge transplantation program forced the implementation of the necessary changes. Since, due to legislative reasons, the EMB procedure is reimbursed only if the patient stays overnight in the hospital, the raised number of HTxs performed will ultimately impact upon the organization of the specific services. Thus the implementation of the Zabrze'18 EMB protocol will help cover all of the diagnostic demands in the cohort of the patients after HTx. To see the general assumptions please refer to Figure 1 depicted below.

\section{Conclusions}

The lack of routinely performed EMBs in the first week and nine months after HTx will decrease the total number of EMBs by $20 \%$ according to the EMB protocol currently used. This feasible option is accessible on the condition of intensive immunosuppression induction with rATG and extremely careful microbiological sensitivity, including anti-bacterial and anti-viral prophylaxis, especially if given in the early post-transplant period.

\section{Acknowledgments}

To Prof. Marian Zembala and Prof. Jan Gummert, the universally renowned pioneers of heart and lung surgery and transplantology in Poland and Germany who fueled the prosperous cooperation between the Silesian Center for Heart Diseases (SCCS) and Herz- und Diabeteszentrum NRW (HDZ NRW).

\section{Conflict of interest}

The authors declare no conflict of interest.

\section{References}

1. Shah KB, Flattery MP, Smallfield MC, et al. Surveillance endomyocardial biopsy in the modern era produces low diagnostic yield for cardiac allograft rejection. Transplantation 2015; 99: 75-80.

2. Orrego CM, Cordero-Reyes AM, Estep JD, et al. Usefulness of routine surveillance endomyocardial biopsy 6 months after heart transplantation. J Heart Lung Transplant 2012; 31: 845-9.

3. Sakakibara S, Konno S. Endomyocardial biopsy. Jpn Heart J 1962; 3: 537-43.

4. Caves PK, Stinson EB, Billingham ME, et al. Serial transvenous biopsy of the transplanted human heart. improved management of acute rejection episodes. Lancet 1974; 1: 821-6.

5. Baraldi-Junkins C, Levin HR, Kasper EK, et al. Complications of endomyocardial biopsy in heart transplant patients. J Heart Lung Transplant 1993; 12: 63-7.

6. Deckers JW, Hare JM, Baughman KL. Complications of transvenous right ventricular endomyocardial biopsy in adult patients with cardiomyopathy: a seven-year survey of 546 consecutive diagnostic procedures in a tertiary referral center. J Am Coll Cardiol 1992; 19: 43-7.

7. Singh V, Mendirichaga R, Savani GT, et al. Comparison of utilization trends, indications, and complications of endomyocardial biopsy in native versus donor hearts (from the Nationwide Inpatient Sample 2002 to 2014). Am J Cardiol 2018; 121: 356-63.

8. Nguyen V, Cantarovich M, Cecere R, et al. Tricuspid regurgitation after cardiac transplantation: how many biopsies are too many? J Heart Lung Transplant 2005; 24 (7 Suppl): S227-31.

9. Barten MJ, Schulz U, Beiras-Fernandez A, et al. A proposal for early dosing regimens in heart transplant patients receiving thymoglobulin and calcineurin inhibition. Transplant Direct 2016; 2: e81.

10. Ansari D, Lund LH, Stehlik J, et al. Induction with anti-thymocyte globulin in heart transplantation is associated with better longterm survival compared with basiliximab. J Heart Lung Transplant 2015; 34: 1283-91. 